more subtle and effective is needed.

Overall, this book is a useful contribution to the literature, and one can hardly disagree with the conclusion that overfishing is "a primary cause of ecosystem disruption". It has some excellent images; in particular figure 17 (shown overleaf), which is a graphic illustration of what it means to fish down the food web, and deserves to be widely reproduced. But I found the book's structure awkward: it has no less than 30 pages of prefaces, and another 30 pages of endnotes, including some substantial discussions of important issues (such as whaling and international institutions), running to several pages each.

Those who work in the field will find that the book is a bold attempt to create an ocean-wide overview that complements the more conventional stock-by-stock reductionist methods. General readers will find a broad, and in places passionate, account of the state of a whole ocean and its resources that is both accurate and informative. One can only hope that it will help to motivate more strenuous and effective efforts to deal with the problems so clearly identified, which is, I am sure, what its authors intend.

John Shepherd is at the Southampton

Oceanography Centre, Empress Dock,

University of Southampton,

Southampton SO14 3ZH, UK.

\section{More on fisheries}

Handbook of Fish Biology and

Fisheries, Vols 1 and 2

edited by Paul J. B. Hart \& John D. Reynolds Blackwell, $£ 130$

\section{New in paperback}

\author{
I Have Landed \\ Stephen Jay Gould \\ Vintage, $£ 7.99$
}

\section{The Constants of Nature \\ by John Barrow \\ Vintage, $£ 8.00$}

"Barrow discusses the role of constants of nature, the historical quest to understand them, the role of the anthropic principle as a guiding philosophy and some recent evidence suggesting that some of the constants of nature are probably not constants at all." Thanu Padmanabhan Nature 419, 780 (2002).

\section{The Borderlands of Science: Where Sense} Meets Nonsense

by Michael Shermer

Oxford University Press, £9.99

The Way of the Cell: Molecules, Organisms and the Order of Life

by Harold M. Franklin

Oxford University Press, £12.95

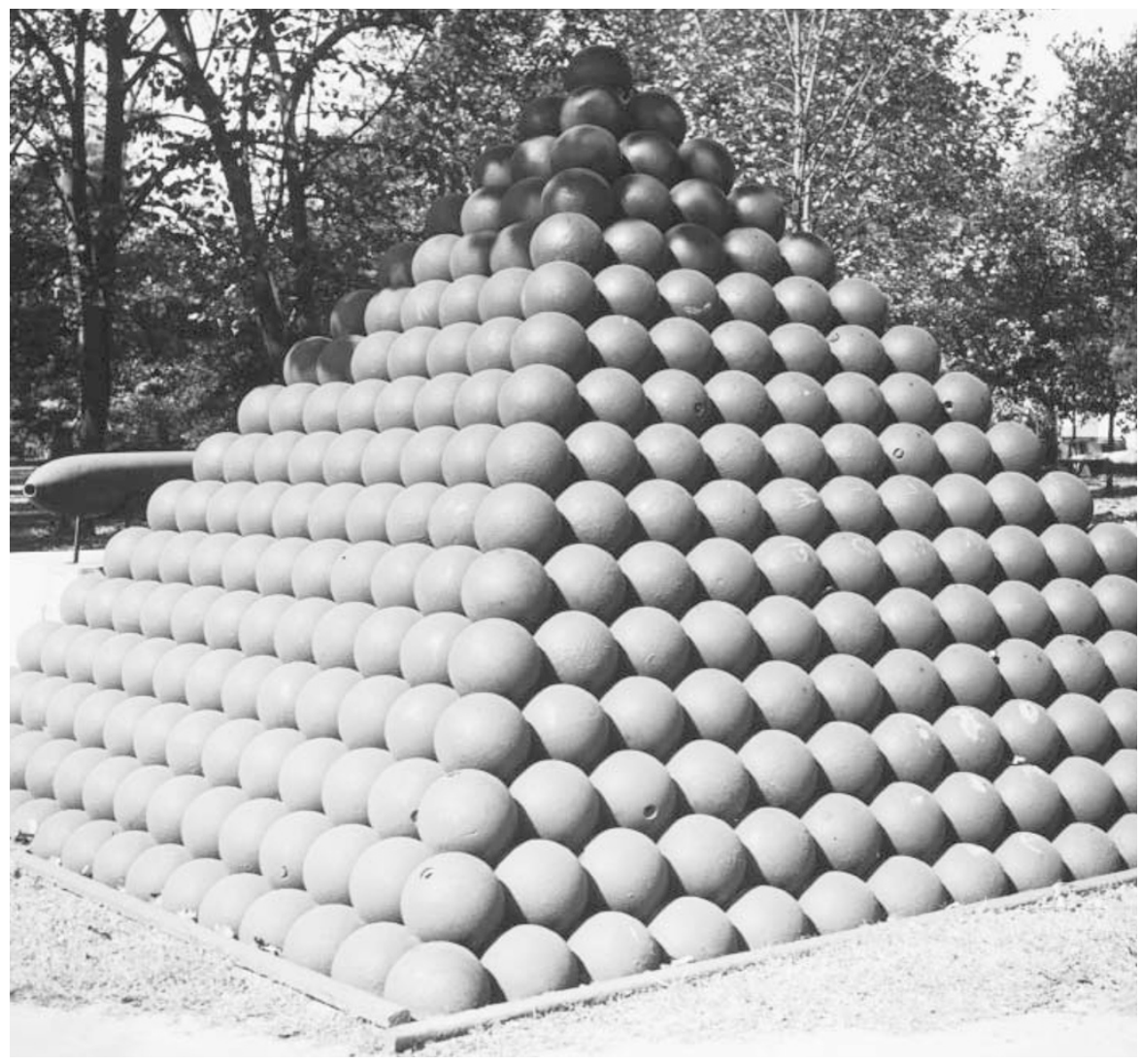

Packing them in: cannon balls stored in a pyramid are stacked together as densely as possible.

• The proof of
the packing

\section{Kepler's Conjecture: How Some of the Greatest Minds in History Helped Solve One of the Oldest Math Problems in the World by George G. Szpiro}

Wiley: 2003. 304 pp. $118.50, \$ 24.95$, €24.95

\section{Neil Sloane}

The classical sphere-packing problem is to determine how densely a large number of identical spheres (such as ball-bearings) can be packed together in a finite space. In 1611 the German astronomer Johannes Kepler stated that no packing could be denser than that of the face-centred cubic (f.c.c.) lattice arrangement favoured by grocers for stacking oranges, which fills about 0.7405 of the available space. It took mathematicians some 400 years to prove him right.

Kepler's Conjecture gives an entertaining and readable account of the history of the problem and the attempts to solve it, culminating with Thomas Hales' successful proof, announced in 1998. George Szpiro also discusses a large number of peripherally related topics, including David Hilbert's list of 23 unsolved mathematical problems from 1900 (Kepler's conjecture is part of problem 18), the kissing-number problem (how many balls can touch another ball of the same size), linear programming and Lord Kelvin's soapfilm problem.

The book is a mixture of mathematics, history and anecdotes. In his research, the author has found many good stories to retell. Even people familiar with the subject will find new anecdotes here, and it seems that most of them are more-or-less true, although one might quibble with the details. Did John Conway's father really teach chemistry to two of the Beatles? Well, sort of.

The tone of the remarks is sometimes derisive, which some readers may find offensive rather than humorous. Young Carl Friedrich Gauss is described as a "little squirt", 'wrangler' is "one of those esoteric blue-ribbon signs of esteem... reserved for British overachievers", and sheaf theory is a "major bore". And after a rather harsh discussion of the attempts of the great Hungarian geometer László Fejes Tóth (his name is consistently misspelled in the book) to prove the dodecahedral conjecture, Szpiro writes: "One might come away from this chapter with the impression that Fejes-Tóth was a bumbling dreamer whose work mostly contained unfulfilled promises and unproven hypotheses. This does not represent the whole picture." Indeed not.

The mathematical content is less satisfactory than the historical part. As William Barlow described in Nature in 1883 (29, 186-188), the f.c.c. packing can be built up by layers. Put down a layer of spheres arranged in a triangular lattice - the arrangement used 
when racking billiard balls - and place another layer on top, and repeat. There are two ways to place subsequent layers. Viewed from above, there are three different positions for the centres of the spheres in any one layer, say A, B and C. If the layers follow the order A, B, C, A, B, C, ..., then the f.c.c. packing is obtained. If they follow the order $\mathrm{A}, \mathrm{B}, \mathrm{A}, \mathrm{B}, \mathrm{A}, \mathrm{B}, \ldots$, then an equally dense packing known as the hexagonal close packing (h.c.p.) is obtained.

Kepler's conjecture is that there are no packings that are denser than the f.c.c. or the h.c.p. packings (or any one of the infinite number of different packings obtained by varying the order of the layers). The f.c.c. and h.c.p. packings have the same density, but they are different: one is a lattice, the other is not. Spiro claims that the f.c.c. and the h.c.p. are "the exact same packing, viewed from different angles". They are not.

Another distraction in the mathematical discussions (which fortunately are set in a different typeface, so they can - and should - be skipped by the casual reader) is the author's misuse of the word 'surface'. Several times he writes of the surface of an object, when he means its area, or even its volume.

One of the oldest theorems about sphere packing was proved by Gauss in 1831, when he showed that the f.c.c. is the densest lattice packing of spheres. Szpiro attempts to reproduce Gauss's proof, but makes a mess of it. For example, on page 255 the determinant needs to be negated, and denoted by a new symbol, $\Delta$, say. Then six occurrences of the letter $D$ on that page need to be changed to $\Delta$. Similar repairs are needed on the next page.

The book hardly mentions one of the main reasons for studying the packing of spheres: its application to digital communications. From the communication theorist's viewpoint, Hales' result on three-dimensional sphere packing is just the beginning of the story. One of the fundamental questions in communication theory is to determine the densest packing of equal balls in multidimensional space. A geometrical way of representing signals, which is at the heart of Claude Shannon's mathematical theory of communication, underlies the high-speed modems that we now take for granted.

Szpiro mentions this subject only briefly, in the final chapter, but the discussion is marred by another error. He describes the following problem as a far-fetched application of packing problems (it is actually a standard type of problem in error-correcting codes). The problem is to find as many strings of ten decimal digits as possible, subject to the constraint that any two of the strings must differ by at least two units in each position. He misuses the known bounds on the density of sphere packing in ten-dimensional space to conclude that "at least $400,000,000$ signals can be represented, which is sufficient for all words in all languages of the globe". However, the correct answer is not 400,000,000, but 5 .

One can only admire Szpiro's valiant attempts to explain the different approaches used by Richard Buckminster Fuller, Wu-Yi Hsiang and Hales in their attacks on the problem (although the serious reader would do better to read Hales' own descriptions). Szpiro's discussion of the arguments between the protagonists is certainly entertaining. He illustrates them with a quotation from Henry Kissinger, who "was once asked why departmental fights are so violent, why back-stabbing is so common among academic colleagues. His answer was short and to the point: 'Because the stakes are so small'" Typically, not quite relevant, but a good story.

As long as readers skip over the technical sections, the book can be recommended as a readable and informative account of a fascinating chapter in the history of geometry.

Neil Sloane is at the AT\&T Shannon Laboratory, 180 Park Avenue, Florham Park, New Jersey 07932-0971, USA.

\section{The rise and fall of the Universe}

\section{Alpha and Omega: The Search for the Beginning and the End of the Universe}

by Charles Seife

Viking Press: 2003.304 pp. $\$ 24.99$

Doubleday: 2003. $£ 18.99$

\section{Peter Coles}

The potentially lucrative market for popular cosmology is pretty crowded these days, so if a book is to be successful it has to stand out from its competitors. One strategy for a publisher is to sign up a professional scientist with something special to say. João Magueijo's Faster Than the Speed of Light (reviewed in Nature 422, 563-564; 2003) and Janna Levin's How the Universe Got its Spots (Weidenfeld $\&$ Nicolson/Princeton University Press, 2002) are two recent books, both written in distinctive, even quirky, styles by specialists for a lay audience. Although very

The collision of gold nuclei at almost the speed of light creates particles in conditions like those just after the Big Bang. different, these books have much in common. Both are insiders' views of the subject, both are highly original because the subject matter is seen from the perspective of the authors' own research, and both include a lot of autobiographical material.

Few scientists are capable of putting their understanding and experiences into words as effectively as these two, so publishers have instead enlisted professional writers to look at the subject from the outside. A science journalist may not have as deep an understanding of the technicalities as a research scientist, but may be more experienced at writing for the general public and consequently better at getting the basic ideas across. Particularly successful examples of this genre are The Whole Shebang by Timothy Ferris (Weidenfeld \& Nicolson/Simon \& Schuster, 1997) and, more recently, Bill Bryson's A Short History of Nearly Everything (reviewed in Nature 424, 725; 2003), which both demonstrate that winners need not necessarily be on the inside track. Sadly, Alpha and Omega by Charles Seife is not among the medal positions.

The book starts promisingly enough, if you can forgive the pseudo-religious overtones of the title (a reference to the Book of Revelations). The suggested emphasis on both the beginning and the end seems a good idea, as there are many books about the birth of the Universe but relatively few about its death. Unfortunately, despite the claims made on the jacket, this theme isn't really taken up by the book itself, except for a few comments in the final chapter. 